\title{
MODELING AND MATHEMATICAL ANALYSIS OF SWARMS OF MICROSCOPIC ROBOTS
}

\author{
Aram Galstyan ${ }^{1}$, Tad Hogg ${ }^{2}$, Kristina Lerman ${ }^{1}$ \\ 1. USC Information Sciences Institute \\ 4676 Admiralty Way \\ Marina del Rey, CA 90292, USA \\ 2. HP Labs \\ 1501 Page Mill Road, MS 1U-19 \\ Palo Alto, CA 94304, USA
}

\begin{abstract}
The biologically-inspired swarm paradigm is being used to design self-organizing systems of locally interacting artificial agents. A major difficulty in designing swarms with desired characteristics is understanding the causal relation between individual agent and collective behaviors. Mathematical analysis of swarm dynamics can address this difficulty to gain insight into system design. This paper proposes a framework for mathematical modeling of swarms of microscopic robots that may one day be useful in medical applications. While such devices do not yet exist, the modeling approach can be helpful in identifying various design trade-offs for the robots and be a useful guide for their eventual fabrication. Specifically, we examine microscopic robots that reside in a fluid, for example, a bloodstream, and are able to detect and respond to different chemicals. We present the general mathematical model of a scenario in which robots locate a chemical source. We solve the scenario in one-dimension and show how results can be used to evaluate certain design decisions.
\end{abstract}

\section{INTRODUCTION}

Swarm Intelligence is a relatively new concept for designing multi-agent systems [4]. Swarms are decentralized systems composed of many simple agents with no central controller. Instead, swarms are self-organizing: constructive collective behavior emerges from local interactions among the agents and between agents and their environment. Selforganization is ubiquitous in nature: Aggregation into multicell organisms in Dictyostelium discoideum colony, trail formation in ants, hive building by bees, and mound construction by termites, are a few examples. The success of these organisms has inspired the design of distributed problemsolving systems.

A major difficulty in designing self-organizing swarms with desired characteristics is understanding the causal relation between individual agent characteristics and collective behavior. One approach is experiments with real agents, e.g., robots, to evaluate behavior under real conditions. However, fabricating enough agents to exhibit swarm behavior is often too expensive or not feasible with current technology. Simulations, such as sensor-based simulations of robots, are usually much faster and less costly than experiments. But these require good models of the robots and their environments, which are often not yet available. Moreover, simulations still require many repetitions to systematically examine consequences of various choices for individual agent behaviors.

Mathematical modeling and analysis offer an alternative to the time-consuming and costly experiments and simulations. Mathematical analysis can be used to study swarm dynamics, predict long-term behavior of even very large systems, and gain insight into system design: e.g., how individual agent characteristics affect the swarm behavior. Such analysis can suggest parameters that optimize group performance, prevent instabilities, etc. These particular choices could then be examined in more detail through simulations or experiments.

This mathematical approach is particularly useful for preliminary evaluation of design trade-offs for robots that cannot yet be built and will operate in poorly characterized environments. As one such application, robots with sizes comparable to bacteria ("nanorobots") could provide many novel capabilities through their ability to sense and act in microscopic environments. Of particular interest are medical applications, where robots and nanoscale-structured materials inside the body could significantly improve disease diagnosis and treatment [9, 19, 20, 15]. Typically, each such robot will have dimensions of a few microns so could readily travel through even the tiniest blood vessels, will be able to communicate with other robots (e.g., using ultrasound) 
over distances of about 100 microns, and will consume in the range of $1-1000$ picowatts.

An example task for such robots operating in the bloodstream is to aggregate and form structures at locations with specific patterns of chemicals. Initially, as robots move passively through the fluid, monitoring for the chemical pattern, they need to avoid disturbing the flow. Thus they will be at low density, i.e., with typical separation between neighboring robots many times larger than their size. On the other hand, to affect the environment, many robots will need to act together (e.g., to block flow in blood vessels feeding populations of cancer cells or to aid in repairing damaged tissues by forming suitable scaffolding). Thus the swarm must change to a higher density of robots, in spite of limited communication ranges. In this denser configuration, communication among neighbors is easier, and simple local controls allow forming a variety of structures either static or dynamically responding to changing forces in the environment, as proposed in the context of large self-reconfigurable robots [3 24]. To quantify this example, a swarm could consist of around $10^{12}$ machines, vastly more than in swarms of larger devices. Even such a large number of machines would have a total mass of only about one gram, and a occupy less than $0.1 \%$ of the typical blood volume of an adult, compared to the $20-40 \%$ occupied by cells.

While fabricating such machines is beyond the ability of current technology, the rapid progress in engineering nanoscale devices should eventually enable their production. For example, ongoing development [12, 10, 18] of molecularscale electronics, sensors and motors provides components for such robots, though these cannot yet be assembled into large numbers of complete systems. Programmable bacteria [25] give large numbers of functioning microscopic systems, and separate DNA computers can respond to Boolean combinations of chemicals in their environment [2]. However relying on biological organisms restricts the robots' material properties and, by using protein synthesis to perform logic operations, limits the computational complexity and speed of control programs.

Beyond the challenge of fabricating the robots is designing and analyzing controls suitable for their physical environments, tasks and capabilities, whose features differ considerably from those of larger robots. Fortunately, we can evaluate control methods prior to building the robots

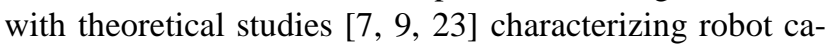
pabilities and their task environments. Moreover, suitable control programs can compensate for some hardware limitations (e.g., limited available power or sensor accuracy). Thus studies of control approaches can identify useful tasks for robots with limited hardware capabilities, as may arise before the fabrication technology is fully mature.

This paper extends the stochastic processes framework developed for modeling teams of larger robots and that has been applied successfully to a number of robotic applications [17]. Our work complements other theoretical studies of microscopic robots [5] 23, 11] by providing a methodology for studying collective swarm behavior. It can also be applied to larger robots that implement chemotaxis as the basis for movement in an external field [6]. While comparison with microscopic experiments must await the technical feasibility of fabricating microscopic robots, our mathematical model can be used to evaluate various design trade-offs for the robot capabilities that can achieve the same swarm performance and can be a useful guide to eventual fabrication by allowing designers to focus on combinations of hardware capabilities easiest to fabricate.

Below we present a general model of the dynamics of a swarm of microscopic robots that are interacting with an external field. In Section 3 we motivate the approach by describing a medically relevant scenario that considers a swarm of microscopic robots moving in a fluid to localize a chemical source. In Section 4 we solve a one-dimensional model and analyze different design choices. We conclude the paper with a discussion of the approach and results.

\section{STOCHASTIC THEORY OF SPATIALLY-DEPENDENT PROCESSES}

Previously, we developed a framework based on stochastic Master Equation for mathematical analysis of homogeneous robotic swarms [13, 16]. Specifically, consider an agent whose controller is an automaton consisting of $L \mathrm{lo}$ cal states. We then assume the overall system is sufficiently well-described by an occupancy vector $\mathbf{n}=\left(n_{1}, n_{2}, \ldots n_{L}\right)$, where $n_{k}$ is the fraction of agents in state $k$. That is, we need only consider the number of agents in each state rather than tracking their individual identities. In designing systems where typical or average behavior is of primary interest, it is useful to characterize the system by a probability distribution $P(\mathbf{n}, t)$, which is the probability the system is in configuration $\mathbf{n}$ at time $t$, and evolves according to the Master Equation [14]. This distribution characterizes the behaviors of an ensemble or multiple independent instances of the swarm. When most such instances have nearly the same behavior, ${ }^{1}$ fluctuations among different instances can be neglected, and the average occupancies $\bar{n}_{k}$ provide a sufficient characterization of the system. The average fraction of agents in state $k$ then evolves according to

$$
\frac{d \bar{n}_{k}}{d t}=\sum_{j} w_{j k} \bar{n}_{j}-\bar{n}_{k} \sum_{j} w_{k j}
$$

\footnotetext{
${ }^{1}$ Consider, for example, a swarm consisting of a large number of agents, each independently executing the same controller. Then, by the law of large numbers, behaviors of different instances of this swarm would be nearly identical.
} 
with $w_{j k}$ the transition rates between the states $j$ and $k$. Note than in general $w_{j k}$ might depend on the fraction of agents in particular states, i.e., average occupancy vector $\overline{\mathbf{n}}$. Eq. 1 governs the evolution of the collective behavior of the system.

While this approach works well for many spatially uniform systems [17], it is too coarse-grained for systems with a spatial correlation in agents' interactions. Thus, it is not sufficient to describe, for example, an ant-like swarm where agents interact through evolving chemical fields or microscopic robots monitoring chemicals released into a fluid. These situations require a generalization of the Master Equation, in which each robot not only has a discrete controller state $k$ but also a continuous coordinate $\mathbf{x}$ (i.e., its spatial location). As with the original formulation, we suppose the number of agents in each state is sufficient to determine the collective behavior of interest. Because $\mathbf{x}$ is a continuous variable, these counts become densities leading us to introduce $\bar{n}_{k}(\mathbf{x}, t)$ as the average robot fraction density in state $k$ at location $\mathrm{x}$ and time $t$. Thus a small volume $\Delta x$ around location $\mathbf{x}$ contains, on average, the fraction $\bar{n}_{k}(\mathbf{x}, t) \Delta x$ of the robots in the system.

Let us consider a system where agents interact with the environment through a certain external chemical field. Let us also assume that agents are able to interact through stigmergy by releasing a special chemical into the environment that we call communicative signal. We denote $\rho(\mathbf{x}, t)$ and $c(\mathbf{x}, t)$ concentration of the chemical and communicative signal, respectively, at point $\mathbf{x}$ at time $t$. Then we can write down the generalized rate equation as follows:

$$
\begin{aligned}
\frac{\partial \bar{n}_{k}(\mathbf{x}, t)}{\partial t} & =\int d \mathbf{x}^{\prime} \sum_{j} w_{j k}\left(\mathbf{x}, \mathbf{x}^{\prime} ; \rho, c\right) \bar{n}_{j}\left(\mathbf{x}^{\prime}, t\right) \\
& -\bar{n}_{k}(\mathbf{x}, t) \int d \mathbf{x}^{\prime} \sum_{j} w_{k j}\left(\mathbf{x}, \mathbf{x}^{\prime} ; \rho, c\right) .
\end{aligned}
$$

Now the transition rates $w_{j k}$ depend not only the state indices $j$ and $k$ and occupation vector but also on the spatial coordinates and concentration of the chemical at the corresponding points. Note also that we have included the dependence of the transition rates on $\mathbf{x}$ and $\mathrm{x}^{\prime}$ explicitly to account for agents' kinematics even in the absence of chemical and communicative concentrations (e.g., to describe freely diffusing agent).

The transition rates $w_{j k}\left(\mathbf{x}, \mathbf{x}^{\prime} ; \rho(\mathbf{x}), c(\mathbf{x})\right)$ summarize the behaviors of the individual robots. For example, the robot's internal state could change when it detects a chemical concentration above a predetermined threshold. In applications of microscopic robots, such detections will often involve a relatively small number of molecules (e.g., on the order of tens) so stochastic fluctuations in the number detected will be a significant source of sensor noise and can be included as a range of new states with nonzero transi- tion rates. Communication among nearby robots, e.g., with ultrasound, allows the robots to reduce noise in estimating chemical gradients and hence perform better than individual robots or bacteria [1], but at a cost of additional power use for the communication. Robot motion, either moving passively with the fluid or using powered locomotion, e.g., to follow chemical concentration gradients, also contributes to the transitions.

While Equation 2 is a general description of the overall system behavior, it is too complex in its present form to be useful. Fortunately, it can be simplified considerably into a more intuitive form by noting that in many physically realistic situations agents' motion can be decoupled from state transitions, so that the transition rate can be represented as

$$
\begin{aligned}
w_{j k} & =\delta_{j k} W_{k}\left(\mathbf{x}, \mathbf{x}^{\prime} ; \rho(\mathbf{x}), \rho\left(\mathbf{x}^{\prime}\right), c(\mathbf{x}), c\left(\mathbf{x}^{\prime}\right)\right) \\
& +\delta\left(x-x^{\prime}\right) w_{j k}(\rho(\mathbf{x}), c(\mathbf{x}))
\end{aligned}
$$

where $\delta_{j k}$ is Kroenecker's symbol ${ }^{2}$ and $\delta(x)$ is its continuous analogue $\delta$-function. In other words, during a transition between two discrete states we neglect the change in robot's position. In Eq. $3 W_{k}$ is an appropriately chosen kernel that describes agents' motion (as index $k$ indicate, it can be different for each state), while the second term describes transition between discrete states.

Equation 3 allows us to separate transition function into terms with purely spatial transitions and terms with purely state transitions. Indeed, using Eq. 3 we can decouple the agents' kinematics from the state transitions between discrete state and rewrite Eq. 2] as follows:

$$
\begin{aligned}
\frac{\partial \bar{n}_{k}(\mathbf{x}, t)}{\partial t} & =\mathcal{L}_{k} \bar{n}_{k}(\mathbf{x}, t)+\sum_{j} w_{j k}(\rho, c) \bar{n}_{j}(\mathbf{x}, t) \\
& -\bar{n}_{k}(\mathbf{x}, t) \sum_{j} w_{k j}(\rho, c)
\end{aligned}
$$

Here $\mathcal{L}_{k}$ is an operator (specified below) that describes the motion of agents in state $k$. The second and third terms in Eq. 4 describe agents state transitions. Note that now $w_{j k}(\rho, c)$ depends on spatial coordinates indirectly, through concentration $\rho(\mathbf{x}, t)$ and $c(\mathbf{x}, t)$. When the concentrations $\rho$ and $c$ are constants, Eq. 1 is recovered by integrating Eq. 4 over the spatial coordinates $\mathbf{x}$, assuming that $\mathcal{L}_{k}$ preserves the number of agents in state $k$ (e.g., no absorbing boundaries) so that integral over the first term in Eq. 4 vanishes.

To specify the operators $\mathcal{L}_{k}$, we note that for the particular environment we are interested in, (i.e., microscopic robots operating in a fluid) robots' motion can be described by a diffusion equation [14]. In this paper, we study chemotactic robots that respond to a chemical and signalling fields

\footnotetext{
${ }^{2}$ Kroenecker's symbol is defined as follows: $\delta_{i j}=1$ if $i=j$ and $\delta_{i j}=0, i \neq j$.
} 
by propelling themselves in the direction of increasing concentration. This capability is modeled after bacterial chemotaxis which allows these single cell organisms to efficiently move towards food sources and away from noxious sources. Although in some cases the exact derivation from the microscopic transition rates is feasible, if very involved (see, for example, [21 8] for treatment of bacterial chemotaxis which can be treated as a biased random walk), chemotaxis in a chemical concentration field $\rho(\mathbf{x}, t)$ is usually introduced into the rate equations phenomenologically by postulating a chemotactic velocity as $V_{D}=\eta_{\rho} \nabla \rho(\mathbf{x}, t)$, where $\eta_{\rho}$ is the so called chemotactic sensitivity (which may itself depend on $\rho$ ). One can then write for operators $\mathcal{L}_{k}$

$$
\mathcal{L}_{k}=D_{k} \nabla^{2}-\mathbf{v} \cdot \nabla-\nabla \cdot\left[\mathbf{V}_{D}^{\rho}(\rho, \nabla \rho)+\mathbf{V}_{D}^{c}(c, \nabla c)\right]
$$

Here, $D_{k}$ is the diffusion coefficient of agents in state $k$ assumed to be a constant, $\mathbf{v}$ is the flow velocity, and $\mathbf{V}_{D}^{\rho}$ and $\mathbf{V}_{D}^{c}$ are the chemotaxis drift velocities of robots due to concentration gradients of the chemical and the communicative signal, respectively.

To proceed further, we should also define how the chemical and concentration fields evolves in time. As an example relevant for microscopic robots, we consider the evolution of this fields in a moving fluid in which the robots operate. The evolutions of $\rho(\mathbf{x}, t) c(\mathbf{x}, t)$ are governed by the diffusion equation:

$$
\begin{aligned}
& \frac{\partial \rho}{\partial t}=D_{\rho} \nabla^{2} \rho-\mathbf{v} \cdot \nabla \rho-\gamma_{\rho} \rho+Q_{\rho}(\mathbf{x}, t) \\
& \frac{\partial c}{\partial t}=D_{c} \nabla^{2} c-\mathbf{v} \cdot \nabla c-\gamma_{c} c+\sum_{k} q_{k} \bar{n}_{k}(\mathbf{x}, t)
\end{aligned}
$$

In Eq. 6 the terms on the right describe, respectively, the diffusion of the chemical (with a diffusion constant $D_{\rho}$ ), the advection of the chemical due to fluid motion with velocity $\mathbf{v}$, the decay of the chemical at rate $\gamma_{\rho}$, and its deposition by sources with intensity profile $Q_{\rho}(\mathbf{x}, t)$. Terms in Eq. 7 have similar meaning, except the deposition rates of signalling chemical is proportional to the fraction of agents in state $k, \bar{n}_{k}(\mathbf{x}, t)$ (note that, generally speaking, the coefficients $q_{k}$ themselves depend on the fraction of agents in state $k$ ). The parameters in this equation could, in general, depend on space and time, as well as the location of the robots (e.g., a sufficiently high concentration of the robots could significantly affect the fluid flow). For simplicity, we will treat them as constants. For microscopic robots, fluid motions will usually be at very low Reynolds number so the fluid flow will be laminar with the velocity $\mathbf{v}$ changing smoothly with location. Viscous forces dominate the motions of such robots with requirements for locomotion mechanisms and power use quite different from experiences with larger robots [22].

Note that the system of Eqs. 4,6 and 7 can be easily generalized to handle multiple chemicals with different dif- fusion coefficients. Another generalization would allow a variable number of agents in the system, in which case we would add a variable for the total number of agents in the system at time $t$ to the variables introduced above describing the fraction in various states.

Equations 477 together with appropriately chosen boundary conditions describe time evolution of the system. In the next section we use this formulation for a prototypical task: finding and localizing at the source of a chemical released into a flowing fluid.

\section{TARGET LOCALIZATION WITH MICROSCOPIC ROBOTS}

Let us consider a D-dimensional volume with multiple targets that release certain chemical into the environment. The task of the microscopic swarm is to aggregate at these targets in order to carry out some actions in the vicinity of the targets. This capability is fundamental to many medical applications envisioned for these microscopic robots. For example, the volume of fluid may be a blood vessel that has been damaged. Robots are required to aggregate at the injury site in order to assist in healing, forming clots, etc.

We consider a simple robot controller that on a high level can be thought to consist of 3 discrete states described below:

State 1 (search): Do a biased random walk in the direction of the communicative signal concentration gradient.

State 2 (communicate): Move towards the chemical source following the concentration gradient of the target chemical and release communicative signal to other robots.

State 3 (disperse): Move away from the target in the direction opposite to the target chemical's concentration gradient for some specified time $\tau$.

To fully specify a robot's behavior, we also have to describe the transitions between these states. The robots start out in State 1, the searching for targets using random diffusive motion and following the gradient of the communicative signal. Once the concentration of the target chemical at a certain point in space is sufficiently high the robot at that point will switch to the State 2: it will start moving towards regions of high concentration (using biased diffusion or gradient following) while releasing a new chemical which acts as a communication signal to attract other robots. With some probability (that can be fixed, or dependent on the concentration of the robots at the source), robots in the State 2 will switch to State 3, where they will disperse from the source, moving in the direction opposite to the gradient. Finally, robots in the State 3 will switch to the searching state with probability $1 / \tau$. The last behavior ensures that 
robots will not be stuck at local maxima of the chemical potential.

Let denote by $n_{1}(\mathbf{x}), n_{2}(\mathbf{x}), n_{3}(\mathbf{x})$ the fraction of robots in each state at point $\mathbf{x}$, with normalization condition

$$
\int d \mathbf{x}\left(n_{1}(\mathbf{x})+n_{2}(\mathbf{x})+n_{3}(\mathbf{x})\right)=1 .
$$

Let $\rho(\mathbf{x})$ and $c(\mathbf{x})$ be the concentrations of the chemical released from the targets and the communicative signal released by robots in State 2 . We also denote by $\mathbf{V}_{D}^{\rho}$ and $\mathbf{V}_{D}^{c}$ the robots' drift velocity in the concentration gradients of chemical (released by the targets) and communicative signal (released by the robots), respectively. Then the set of equations describing the evolution of the system is as follows:

$$
\begin{aligned}
\frac{\partial n_{1}}{\partial t} & =D_{1} \nabla^{2} n_{1}-\mathbf{v} \cdot \nabla n_{1}-\nabla \cdot\left[\mathbf{V}_{D}^{c} n_{1}\right] \\
& -n_{1} F(\rho)+\frac{n_{3}}{\tau} \\
\frac{\partial n_{2}}{\partial t} & =D_{2} \nabla^{2} n_{2}-\mathbf{v} \cdot \nabla n_{2}-\nabla \cdot\left[\mathbf{V}_{D}^{\rho} n_{2}\right] \\
& +n_{1} F(\rho)-G\left(n_{2}, \rho, c\right) n_{2} \\
\frac{\partial n_{3}}{\partial t} & =D_{3} \nabla^{2} n_{3}-\mathbf{v} \cdot \nabla n_{3}+\nabla \cdot\left[\mathbf{V}_{D}^{\rho} n_{3}\right] \\
& +G\left(n_{2}, \rho, c\right) n_{2}-\frac{n_{3}}{\tau}
\end{aligned}
$$

where $F(\rho)$ is the concentration-dependent transition rate from State 1 to State $2, G\left(n_{2} ; \rho ; c\right)$ is the transition rate from State 2 to State 3 , and $1 / \tau$ is the probability that a robot in State 3 will switch to State 1.

Equations 8, 10 have a simple intuitive interpretation. The first two terms in Eq. 8] describe robots motion in State 1: diffusive searching and following communicative signal, if present. The third term describes the drift in the flow. The fourth term describes transitions to State 2 at the rate $F(\rho)$, which depends on the concentration of the target field. The last term describes transition of robots from State 3 to State 1 after the robots have moved in the direction opposite to the concentration gradient for a period of time $\tau . G\left(n_{2}, \rho, c\right)$ is the rate at which robots transition from State 2 to State 3, and it could in principle depend on the local concentrations of the gradients, as well as the number of agents present at the target site, for example, when presence of a certain minimum number of robots is required for executing an action.

We have to complement these three equations with two more to account for the evolution of chemicals $\rho$ and $c$, that are obtained from Equations ??,?? and ?? as follows:

$$
\begin{aligned}
\frac{\partial \rho}{\partial t} & =D_{\rho} \nabla^{2} \rho-\mathbf{v} \cdot \nabla \rho+\sum_{i=1}^{M} Q_{i} \delta\left(\mathbf{x}-\mathbf{x}_{i}\right)-\gamma_{\rho} \rho \\
\frac{\partial c}{\partial t} & =D_{c} \nabla^{2} c-\mathbf{v} \cdot \nabla c+q_{c} n_{2}-\gamma_{c} c
\end{aligned}
$$

In Eq. $11 \mathbf{x}_{i}$-s, $i=1,2, . . M$ are the locations of the target sources, $Q_{i}$ is the intensity of source $i$, and $\gamma_{\rho}$ is the decay rate of the target chemical. Similarly, in Eq. 12 $q_{c}$ is the intensity of communication signal released by a robot in State 2 , while $\gamma_{c}$ is the decay rate of the signal.

\section{RESULTS FOR A SIMPLIFIED 1D SCENARIO}

In this section we present results for a $1 D$ geometry and a single target scenario. We consider the case when the liquid flow is very slow compared to other time scales so we can set $\mathbf{v}=0$. Also, since there is only one target, we neglect the third (dispersing) behavior so that two possible states are State 1 ("search") and State 2 ("communicate"). The target is located at $x=1$ and serves as a point source for the chemical. We assume that the diffusion of the chemical happens much faster compared to robots' diffusion, and it quickly reaches its steady state profile. Hence, the equation for evolution of $\rho(x, t)$ can be solved separately, with a solution

$$
\rho(x, \infty) \equiv \rho(x)=Q_{0} e^{-\sqrt{\gamma_{\rho} / D_{\rho}}(1-x)}, 0 \leq x \leq 1 .
$$

For the results presented here we used $Q_{0}=0.1, D_{\rho}=0.2$ and $\gamma_{\rho}=0.5$.

All robots start at State 1 and are initially localized at $x=0$. We assume that a transition from State 1 to State 2 happens whenever a robot in State 1 detects the target's chemical above a certain threshold level $\rho_{0}$, so that the transition rate is $F(\rho)=\theta\left(\rho-\rho_{0}\right)$, where $\theta(x)$ is the step function, $\theta(x)=1$ if $x \geq 0$ and $\theta(x)=0, x<0$. While in State 2, robots move in the chemical gradient with a constant drift velocity $V_{D}$ and release a communicative signal with intensity $q_{c}$.

To proceed further, we need to specify the dependence of the drift velocity in State 1 on the concentration of communicative signal $c$. Again, we assume that once a robot detects communicative signal above certain threshold $c_{0}$, it propels itself through the fluid in the direction of the gradient with a constant drift velocity $V_{D}$. Then the dynamics of the system is described by the following system of equations:

$$
\begin{aligned}
\frac{\partial n_{1}}{\partial t} & =D_{n} \frac{\partial^{2} n_{1}}{\partial x^{2}}-V_{D} \theta\left(c-c_{0}\right) \frac{\partial n_{1}}{\partial x}-F(\rho) n_{1} \\
\frac{\partial n_{2}}{\partial t} & =D_{n} \frac{\partial^{2} n_{2}}{\partial x^{2}}-V_{D} \frac{\partial n_{2}}{\partial x}+F(\rho) n_{1} \\
\frac{\partial c}{\partial t} & =D_{c} \frac{\partial^{2} c}{\partial x^{2}}+q_{c} n_{2}-\gamma_{c} c
\end{aligned}
$$

To study the effect of different design parameters on aggregation behavior of the robots at the target, we solved the system Eq. 14-16 numerically. We used the following parameters (in dimensionless units): $D_{n}=0.01, D_{c}=0.05$, 
$V_{D}=0.1, q_{c}=0.1, \gamma_{c}=0.01$. For the detection thresholds we used $c_{0}=0.001$ and $\rho_{0}=0.01$, the later assuring that that robots detect the chemical approximately midway in the interval $[0,1]$. We used reflective boundary conditions for $n_{1}$ and $n_{2}, \partial n_{1} /\left.\partial x\right|_{0,1}=\partial n_{2} /\left.\partial x\right|_{0,1}=0$, and absorbing boundary conditions for $c, c(0)=c(1)=0$.

In Fig.11we plot the spatia-temporal evolution of robots' densities with and without communication. Clearly, the density peak at $x=1$ is stronger for the system with communicative behavior. This suggests that communication indeed helps the robots to aggregate better. In addition, the aggregation process with communication happens faster than without communication. This is also shown in Fig. 2] where we plot the density of robots at $x=1$ as a function of time for three different cases: free diffusion ${ }^{3}\left(V_{D}=0\right)$, gradient following without communication $\left(V_{D} \neq 0, q_{c}=0\right)$, and gradient following with communication $\left(V_{D}, q_{c} \neq 0\right)$. As it can be seen from Fig. 2 the systems with gradient following and communicative behavior do demonstrate aggregative behavior, and it is more pronounced for the system with communication. For instance, at time $t=10$ the robot density at $x=1$ and with communication is more than 3 times higher than in the non-communicating case.

One of the design objectives is to have robots aggregate at the target fast enough, while at the same time not dissipating too much power due to the propelling. To examine this tradeoff, let us consider the dependence of the aggregation time (defined as time needed for fraction $n_{0}$ of robots to reach the vicinity of the target which we define as the interval $[0.95,1]$,) on the drift velocity $V_{D}$. In Fig. 3 we plot aggregation time vs $V_{D}$ for three different values of $n_{0}$. One observes that if increasing the drift velocity from $V_{D}=0$, the aggregation time decreases monotonically, with a steeper decline for larger $n_{0}$. However, it soon "saturates", so that increasing $V_{D}$ further has very small effect on the aggregation time. This is because for large values of $V_{D} / D_{n}$, the aggregation time is mainly dominated by time required for robots to diffuse and detect chemical gradient, and increasing $V_{D}$ clearly does not have any effect on this time. Hence, depending on the desired number of robots in the vicinity of the target, as well as the required aggregation time, the best strategy for robots might be to have a moderate drift velocity. Note that this type of analysis can be used to assess the energy-efficiency of various behaviors since power required to propel a robot through a fluid with velocity $V_{D}$ scales with $V_{D}$.

\footnotetext{
${ }^{3}$ Note that the absence of aggregation for free diffusing robots is due to reflective boundary conditions at the source for $n_{1}$ and $n_{2}$. If one employs absorbing boundary conditions instead, robots will demonstrate aggregative behavior even with free diffusion.
}

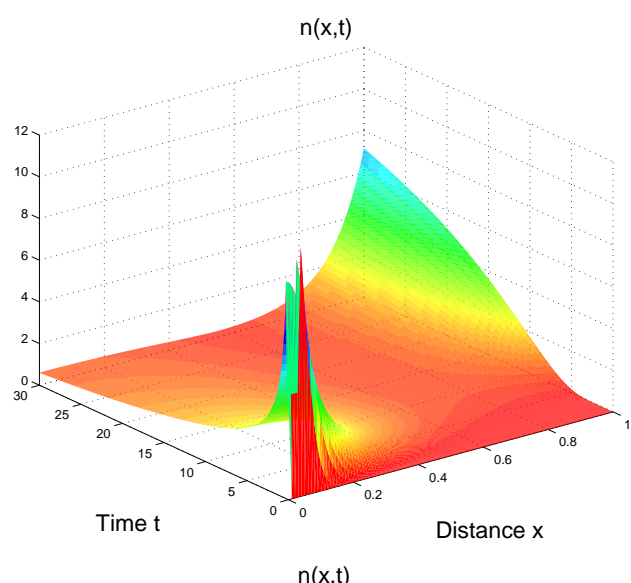

(a)

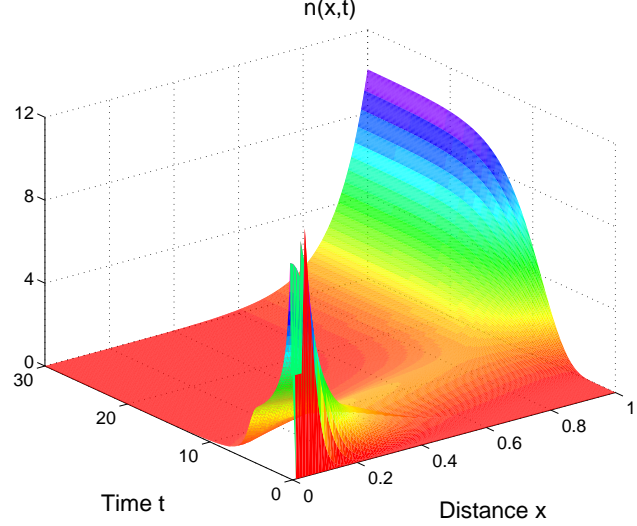

(b)

Figure 1. Time evolution of robot densities without communication (a) and with communication (b)

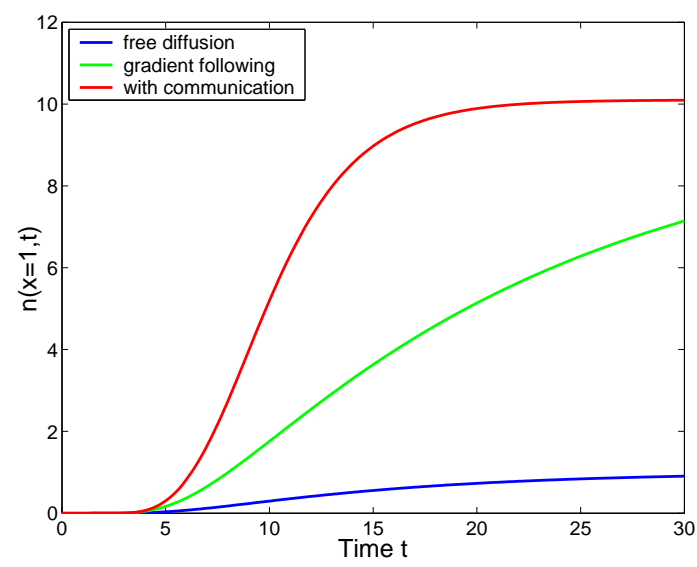

Figure 2. Time evolution of robot densities at $x=1$ for three different strategies 


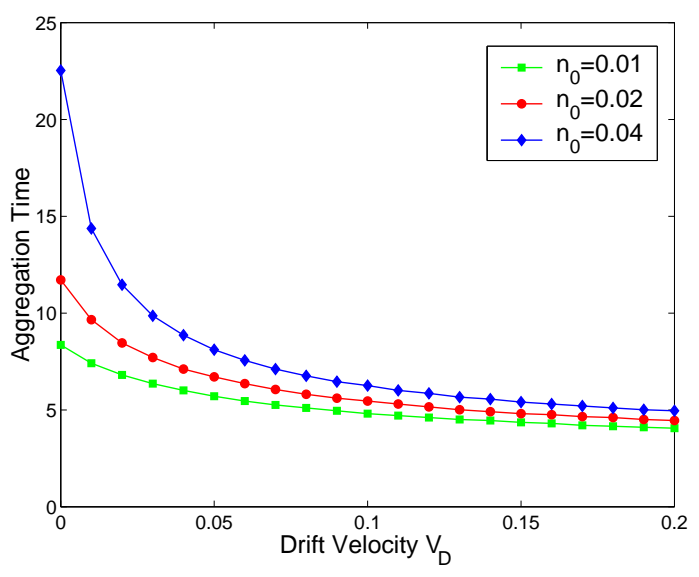

Figure 3. Aggregation time as a function of drift velocity, for three different values of $n_{0}$.

\section{DISCUSSION}

Microscopic robots face physical environments quite different from our experience with larger robots. In particularly, many interesting tasks for such robots will involve interaction with spatially varying fields, such as chemical concentrations. In this paper, we showed how to incorporate such fields into a previously proposed approach relating individual robot behaviors to that of the population as a whole. The analytic framework readily incorporates Brownian motion and viscous fluid flow. The framework also applies to macroscopic robots programmed to emulate behavior of microscopic organisms - for example, chemotaxis [6].

As future work, it would be interesting to examine more realistic scenarios, involving three-dimensional fluid flow in vessels with quantitatively accurate physical parameters for the environment and robot sensors and actuators. We could also compare alternate approaches to various robot capabilities, e.g., acoustic vs. chemical communication. For instance, with more extensive use of communication, nearby robots could compare concentration measurements to better estimate concentration gradients, thereby introducing a spatial correlation in the state changes. Our approach applies to these more complicated physical scenarios, but becomes more computationally demanding to solve numerically. Nevertheless, the computation cost involved to identify useful design trade-offs through our approach will be less than that involved with detailed simulations (especially for tasks involving large populations of robots). Actual experiments with physical devices will be even more challenging.

Limitations of this approach are its underlying assumptions, namely that to determine relevant collective swarm behaviors the occupancy numbers are a sufficient description of the individual robots and fluctuations are small so the averages over many independent instances of the swarm are close to the actual observed behaviors in most of those instances. More broadly, this approach connects average, aggregate behavior with local robot controllers rather than providing specific details of individual robots. For applications involving large populations of microscopic robots, the law of large numbers will ensure behaviors are usually close to average.

\section{REFERENCES}

[1] J. P. Adler. Chemotaxis in bacteria. Science, 153:708-716, 1966.

[2] Yaakov Benenson, Binyamin Gil, Uri Ben-Dor, Rivka Adar, and Ehud Shapiro. An autonomous molecular computer for logical control of gene expression. Nature, 429:423-429, 2004.

[3] Hristo Bojinov, Arancha Casal, and Tad Hogg. Multiagent control of modular self-reconfigurable robots. Artificial Intelligence, 142:99-120, 2002. Available as arxiv.org preprint cs.RO/0006030.

[4] Eric Bonabeau, Marco Dorigo, and Guy Theraulaz. Swarm Intelligence: From Natural to Artificial Systems. Oxford University Press, New York, 1999.

[5] Adriano Cavalcanti and Tad Hogg. Simulating nanorobots in fluids with low Reynolds number. In Proc. of the 11th Foresight Conference on Molecular Nanotechnology, October 2003 .

[6] Amit Dhariwal, Gaurav S. Sukhatme, and Aristides A. G. Requicha. Bacterium-inspired robots for environmental monitoring. In Proceedings of the 2004 IEEE Intl. Conference on Robotics and Automation, New Orleans, LA. IEEE Press, April 2004.

[7] K. Eric Drexler. Nanosystems: Molecular Machinery, Manufacturing, and Computation. John Wiley, NY, 1992.

[8] Radek Erban and Hans G. Othmer. From individual to collective behavior in bacterial chemotaxis. SIAM J. Applied Math, 65(2):361-391, 2004.

[9] Robert A. Freitas Jr. Nanomedicine, volume 1. Landes Bioscience, Georgetown, TX, 1999. Available at www.nanomedicine.com.

[10] J. Fritz et al. Translating biomolecular recognition into nanomechanics. Science, 288:316-318, 2000.

[11] V. Gazi and K. M. Passino. Stability analysis of social foraging swarms. IEEE Trans. on Systems, Man and Cybernetics, B34:539-557, 2004.

[12] Joe Howard. Molecular motors: Structural adaptations to cellular functions. Nature, 389:561-567, 1997.

[13] Bernardo A. Huberman and Tad Hogg. The behavior of computational ecologies. In B. A. Huberman, editor, The Ecology of Computation, pages 77-115, Amsterdam, 1988. Elsevier (North-Holland). 
[14] N. G. Van Kampen. Stochastic Processes in Physics and Chemistry. Elsevier Science, Amsterdam, revised and enlarged edition, 1992.

[15] Balazs L. Keszler, Istvan J. Majoros, and James R. Baker Jr. Molecular engineering in nanotechnology: Structure and composition of multifunctional devices for medical application. In Proc. of the Ninth Foresight Conference on Molecular Nanotechnology, 2001.

[16] Kristina Lerman and Aram Galstyan. Two paradigms for the design of artificial collectives. In Kagan Tumer and David Wolpert, editors, Collectives and Design of Complex Systems, pages 231-256. Springer Verlag, New York, 2004.

[17] Kristina Lerman, Alcherio Martinoli, and Aram Galstyan. A review of probabilistic macroscopic models for swarm robotic systems. In Sahin E. and Spears W., editors, Swarm Robotics Workshop: State-of-the-art Survey, number 3342 in LNCS, pages 143-152. Springer-Verlag, Berlin Heidelberg, 2005.

[18] Carlo Montemagno and George Bachand. Constructing nanomechanical devices powered by biomolecular motors. Nanotechnology, 10:225-231, 1999.

[19] Kelly Morris. Macrodoctor, come meet the nanodoctors. The Lancet, 357:778, March 102001.

[20] NIH. National Institutes of Health roadmap: Nanomedicine, 2003. Available at http://nihroadmap.nih.gov/nanomedicine/index.asp.

[21] Hans G. Othmer and Thomas Hillen. The diffusion limit of transport equations ii: Chemotaxis equations. SIAM J. Applied Math, 62(4):1222-1250, 2002.

[22] E. M. Purcell. Life at low Reynolds number. American Journal of Physics, 45:3-11, 1977.

[23] Aristides A. G. Requicha. Nanorobots, NEMS and nanoassembly. Proceedings of the IEEE, 91:1922-1933, 2003.

[24] B. Salemi, W.-M. Shen, and P. Will. Hormone controlled metamorphic robots. In Proc. of the Intl. Conf. on Robotics and Automation (ICRA2001), 2001.

[25] Ron Weiss and Thomas F. Knight, Jr. Engineered communications for microbial robotics. In Proc. of Sixth Intl. Meeting on DNA Based Computers (DNA6), 2000. 